Article original

\title{
Epidémiologie générale du diabète de type 1 chez l'enfant: Le registre de la wilaya d'Oran 1973-2017
}

\author{
General epidemiology of type 1 diabetes in children: The diabetes register of the \\ department of Oran 1973-2017
}

\author{
Mahmoud Touhami', Assia Bouchetara², Amel Zennaki', Malika Naceur', Asma Aoui', Mounia \\ Gharnouti ${ }^{3}$, Charef Latroch', Karim Bouziane-Nedjadi'. \\ 'Service de pédiatrie « $\mathrm{C}$ » $\mathrm{CHU}$ d'Oran \\ 2Service d'infectiologie pédiatrique EHS Canastel \\ ${ }^{3}$ Service de pédiatrie HRMU d'Oran.
}

Auteur correspondant: mahmoud_touhami@yahoo.fr

Article reçu le 20/09/2018/Accepté le 20/10/2018

\section{MOTS CLÉS \\ Epidémiologie, inci- dence, DT1, enfant, registre, wilaya d'Oran.}

\section{Résumé}

Introduction - Le diabète chez l'enfant subit une mutation profonde en termes de fréquence et de recul de l'âge de début depuis une vingtaine d'années au niveau mondial. L'Algérie est particulièrement impactée par l'épidémie au point de figurer depuis 2016 dans le 'top 10' des pays à très forte incidence de diabète de type 1 (DT1). Notre étude a pour but de présenter l'évolution épidémiologique du DT1 de l'enfant à travers le registre de la wilaya d'Oran.

Matériels et méthodes - La population de référence est celle de la wilaya d'Oran. Les nouveaux cas de DT1 proviennent des unités de première ligne et des services de pédiatrie. L'enregistrement était centralisé au niveau du service de pédiatrie «C» du CHU d'Oran depuis 1973 avec une exhaustivité proche de 100\%.

Résultats - Du 1er janvier 1973 au 31 décembre 2017, 2358 nouveaux cas de DT1 de moins de 15 ans au diagnostic du DT1 ont été enregistrés. L'incidence annuelle moyenne des moins de 15 ans des cinq dernières années 2013-2017 s'établit à $31,12 \pm 3,60$ cas pour 100000 avec une évolution annuelle moyenne de $12,78 \%$ sur les 25 dernières années. Les évolutions annuelles moyennes des classes d'âge pédiatriques de [0-4], [5-9] ans et [10-14] ans sont de 15,03, 15,50 et de 9,10\%, respectivement. L'incidence pour 1000 naissances vivantes des années 1998-2002 montre un risque de contracter un DT1 dans les 15 premières années de vie de 3,5 p. 1000. La prévalence était de 207 pour 100.000, soit 1 DT1 pour 482 enfants de moins de 15 ans, au 31 décembre 2017. Le sex-ratio s'établit à 0,99 pour l'ensemble des cas, mais subit des variations cycliques significatives avec des extrêmes de 0,80 et 1,14 $(\mathrm{p}=0,02)$. La saisonnalité hiver/été, significative de 1973 à 2014 en faveur des mois d'hiver, ne l'est plus à partir de 2015. 
Conclusion - L'augmentation persistante de l'incidence et le rajeunissement marqué de l'âge d'apparition du DT1 de l'enfant de la wilaya d'Oran sont une réalité depuis une vingtaine d'années. Cette évolution, liée essentiellement à l'environnement, ne va pas sans poser de nouvelles difficultés aux familles et aux équipes en charge.

\section{KEY WORDS}

Epidemiology, incidence, T1D, child, diabetes registry, Algeria.

\section{Introduction}

Le diabète de type 1 chez l'enfant subit une mutation profonde depuis une vingtaine d'années au niveau mondial. Le changement touche essentiellement à l'incidence des moins de 15 ans sous la forme d'une véritable épidémie, et à l'âge d'apparition de plus en plus précoce posant de sérieux problèmes en matière de prise en charge en milieu pédiatrique [1].

\section{Abstract}

Introduction - Diabetes in children is undergoing a profound change in terms of frequency and decrease in age in the last twenty years worldwide. Algeria is particularly impacted by the epidemic to the point of appearing in the 'top 10' 2016 of countries with very high incidence of type 1 diabetes (T1D). Our study aims to present the epidemiological evolution of T1D in children through a recruitment of 45 years in the register of the department of Oran.

Materials and Methods - The reference population is that of the department of Oran. New cases of T1D are children under 15 years old coming from front-line units and pediatric departments. Registration was centralized at the level of the « $\mathrm{C}$ » pediatric unit of Oran University Hospital since 1973 with an assessment close to $100 \%$.

Results - From January 1st, 1973 to December 31st, 2017, 2358 T1D new cases aged less than 15 years at T1D diagnosis have been registered. The average annual incidence under 15 years old for the last 5 years $2013-2017$ is $31.12 \pm 3.60$ cases per 100 000 with an annual evolution average of $12.78 \%$ in the last 25 years. The annual evolution average of pediatric age groups are $15.03,15.50$ and $9.10 \%$, respectively for children of [0-4], [5-9] and [10-14] years old. The incidence per 1000 live births shows a risk of contracting T1D in the first 15 years of life of around 3.5. At December 31, 2017, the prevalence was 207 for 100000 equivalent to 1 T1D for 482 children under 15 ,. The sex-ratio for all cases is 0.99 (1175 boys / 1183 girls), but undergoes cyclical variations with extremes of 0.80 and 1.14 between the periods 1978-1997 and 1998$2007(p=0.02)$. The winter/summer seasonality, significant from 1973 to 2014 in favor of winter months is no longer available starting from 2015.

Conclusion - In agreement with other teams, we confirm the outsized increase in the incidence of T1D and the rejuvenation of the age at onset in children under 15 in our country. This evolution, mainly related to environmental problems poses new difficulties to families and teams in charge of the disease.
L'Algérie, qui n'échappe pas à cette évolution, est de plus particulièrement impactée par l'épidémie au point de figurer dans le 'top 10' des pays à très forte incidence de DT1, avec des chiffres annuels de 26 pour 100000 enfants et adolescents de moins de 20 ans et de 2900 nouveaux cas de moins de 15 ans, selon l'Atlas 2017 du Diabète de la Fédération International du Diabète (FID) [2] dont les estimations ont été produites par les chercheurs de la Queen's University de Belfast [3]. 
Notre étude a pour but de présenter l'évolution épidémiologique du DT1 de l'enfant à travers un recrutement privilégié de 45 années faisant l'objet du registre de la wilaya d'Oran.

\section{Matériels et méthodes}

La population de référence est celle de la wilaya d'Oran. Les chiffres retenus sont ceux de l'Office National des Statistiques d'après les recensements généraux de 1977, 1987, 1998 et 2008 et ceux déduits par projections intercensitaires pour les années intermédiaires tenant compte des taux de croissance. En 2015, la wilaya d'Oran comptait 1.800 .000 habitants. Les moins de 15 ans en représentaient $28,1 \%$, les moins de 5 ans $11,2 \%$, les [5-9] ans $8,7 \%$ et les [10-14] ans $8,2 \%$. Le nombre de naissance a été de 41.000 la même année.

Les nouveaux cas de DT1 provenaient des unités publiques de soins primaires, des cabinets de praticiens libéraux, du CHU et des hôpitaux périphériques de la wilaya d'Oran. L'enregistrement était centralisé au niveau du Service de Pédiatrie «C» du CHU d'Oran. Tous les patients retenus répondaient aux critères du DT1, tels qu'ils ont été adoptés par les organismes internationaux [4,5]. La notion de l'insulinodépendance a été retenue sur l'amaigrissement, la cétose, parfois l'acidocétose initiale et sur la dépendance permanente vis-à-vis du traitement insulinique.

Ont été exclus de l'analyse générale les sujets présentant d'autres formes de diabète chez l'enfant comme le DT2, émergeant chez l'enfant, et les diabètes plus rares tels que le diabète induit, le syndrome de Wolfram, le syndrome de Rogers et les diabètes mono-géniques. La source secondaire était représentée par les données de la caisse d'assurance sociale de la wilaya d'Oran. L'enregistrement a été continu et exhaustif depuis 1973. Nous pouvons considérer que les données pour la wilaya d'Oran, ont été validées avec une exhaustivité proche de $100 \%$. La saisie informatique a évité les doublons.

Pour le calcul de l'incidence, ont été retenus tous les enfants dont le DT1 à débuté entre le $01 / 01 / 1973$ et le $31 / 12 / 2017$, âgés de moins de 15 ans au moment du diagnostic et résidant dans la wilaya d'Oran depuis plus de 6 mois. Le début du DT1 correspondait au jour de la première injection d'insuline. L'incidence a été exprimée en nombre de nouveaux cas, pour 100000 enfants.

L'incidence annuelle moyenne ressort du total des nouveaux cas par période quinquennale, afin de limiter l'effet des fluctuations annuelles. Les incidences par classe d'âges pédiatriques [0-4], [5-9] et [10-14] ans ont été exprimées en moyenne annuelle du total des cas relevés dans les périodes intercensitaires, les recensements indiquant précisément les proportions relatives des classes d'âge. Pour le calcul de la prévalence ont été retenus tous les enfants atteints de DT1 vivants, résidant à l'intérieur des limites géographiques de la wilaya d'Oran et âgés de moins de 15 ans au 31/12/2017. La prévalence a été exprimée pour 100000 enfants de moins de 15 ans et pour chacune des trois classes d'âge pédiatriques de 5 en 5 ans.

La statistique fait appel aux comparaisons de moyennes et de pourcentages pour les distributions normales et au test de Wilcoxon en cas de distribution non paramétrique.

\section{Résultats}

Du 1er janvier 1973 au 31 décembre 2017, 2358 nouveaux cas de DT1 âgés de moins de 15 ans au diagnostic ont été enregistrés dans la wilaya d'Oran.

L'évolution de l'incidence annuelle moyenne par périodes quinquennales et pour 100000 enfants de moins de 15 ans est représentée sur la figure 1. Elle aboutit pour la période 2013-2017 à un chiffre de 31,12 $\pm 3,60$ (IC 95\%, 27,90-34,23) et l'évolution annuelle moyenne s'établit à $12,78 \%$ pour les 25 dernières années.

Figure 1. Incidence du DT1 de 1973 à 2017 en moyennes annuelles quinquennales p.100.1000 enfants de moins de 15 ans. Registre de la wilaya d'Oran, $\mathrm{n}=2358$.

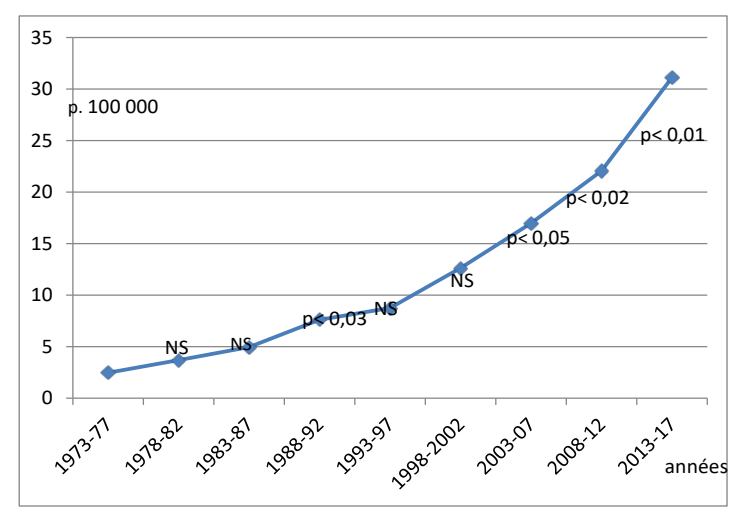

L'évolution des incidences selon les classes d'âge pédiatriques exprimées en moyennes par périodes centrées sur les recensements fait l'objet de la figure 2 . Pour la période

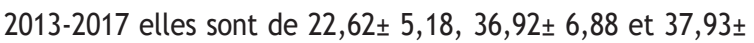
6,53 respectivement pour les classes [0-4], [5-9] et [10-14] ans. Leurs évolutions annuelles moyennes pour les 25 dernières années s'établissent à $15,03,15,50$ et $9,10 \%$, respectivement. 
Figure 2. Incidences annuelles moyennes du DT1par classes d'âges pédiatriques et pour 100.1000 enfants. Registre de la wilaya d'Oran, $n=2358$.

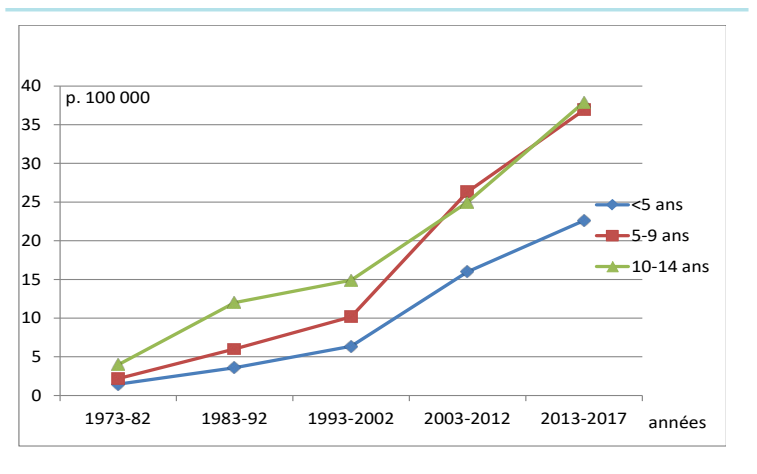

La figure 3 montre le recul de l'âge d'apparition du DT1 sous la forme d'une bascule significative des incidences par classes d'âge qui se produit dès l'année 2010. La moyenne

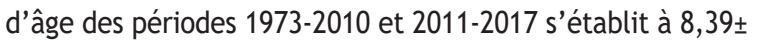

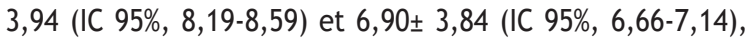
respectivement $(p<10-9)$. On relève que les âges de début les plus représentés depuis 2010 vont de la 4ème à la 11ème année, avec un pic à la 7ème, sont différents de ceux de la période d'avant 2011 où les âges les plus touchés allaient de la 11ème à la 14ème année. Par ailleurs, on ne retrouve pas de différences notables de l'âge de début en fonction du sexe avec toutefois un excès de filles les 1ère, 2ème et 12 ème années et un excès de garçons les 3èmes, 4ème et 15ème années.

Figure 3. Ages de début du DT1 de l'enfant de la wilaya d'Oran atteint de DT1. Evolution des proportions relatives des 3 classes pédiatriques avant et après l'année $2010(n=2358)$

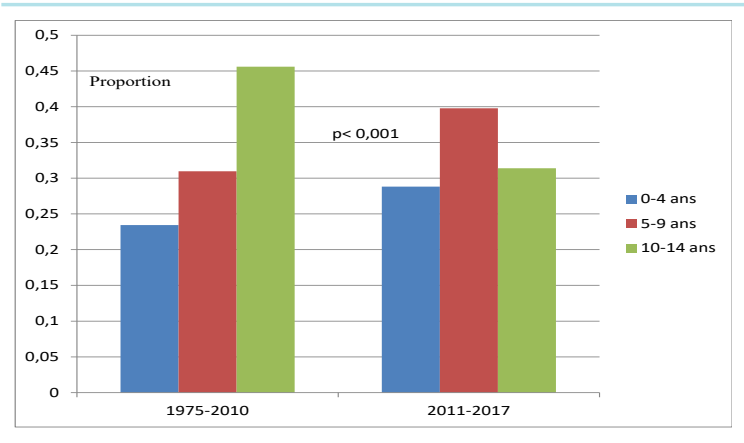

La figure 4 montre la progression du nombre de cas de DT1 diagnostiqués avant l'âge de 15 ans après report sur leur année de naissance. Le calcul pour 1000 naissances vivantes effectué pour les années de naissance 1998-2002 montre un risque de contracter un DT1 dans les 15 années suivantes de l'ordre de 3,5 pour 1000 .
Figure 4.DT1 de l'enfant de la wilaya d'Oran. Cas par incidents par année de naissance

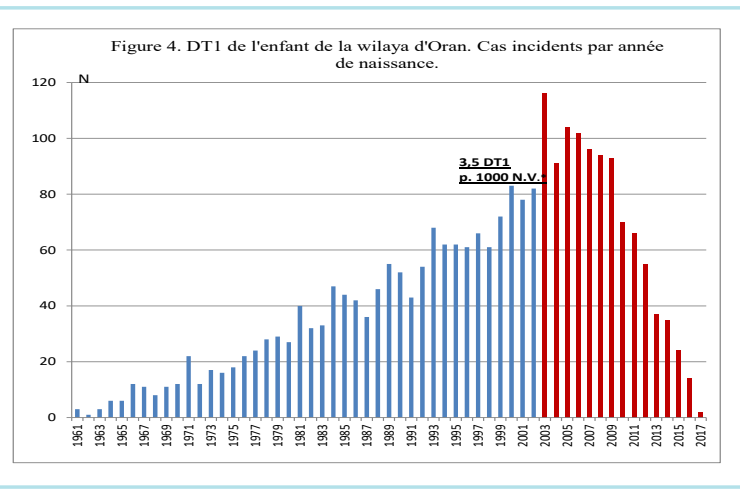

La prévalence était de 207 pour 100000 , soit 1 DT1 pour 482 enfants de moins de 15 ans au 31 décembre 2017. Le tableau 1 détaille les prévalences par classes d'âge pédiatriques de 5 en 5 ans.

Le sex-ratio (Garçons/Filles.) s'établit pour l'ensemble des cas à 0,99 (1175/1183), mais subit des variations cycliques significatives avec des extrêmes de 0,80 dans la période 1988-1997 et de 1,14 dans la période 1998-2000 ( $p=0,02$ ). Le tableau 2 détaille l'évolution du sex-ratio par périodes quinquennales.

Tableau 1. Prévalence du DT1 de l'enfant de moins de 15 ans de la wilaya d'Oran au 31.12.2017

\begin{tabular}{|c|c|c|c|}
\hline Classe d'âge & $\begin{array}{c}\text { Nombre de DT1 } \\
\text { vivants au } \\
31.12 .2017\end{array}$ & $\begin{array}{c}\text { Nombre de } \\
\text { DT1/100.000 } \\
\text { enfants }\end{array}$ & 1 DT1 pour... \\
\hline $0-4$ ans & 112 & 59 & $\ldots 1705$ enfants \\
\hline $5-9$ ans & 378 & 242 & $\ldots . .412$ enfants \\
\hline $10-14$ ans & 509 & 377 & $\ldots .265$ enfants \\
\hline $0-14$ ans & 999 & 207 & $\ldots . .482$ enfants \\
\hline
\end{tabular}

La saisonnalité comparée hiver/été, significative entre mois froids et mois chauds de 1973 à $2014(p<0,01)$ disparaît en 2015-2017 $(p=0,58)$. De même que la proportion hiver/été des 2 périodes considérées se révèle différente avec $58,69 \%$ des cas durant les hivers $1973-2014$ contre $52,78 \%$ en 2014 $2017(p<0,05)$. Les tableaux 3 et 4 illustrent ces faits.

\section{Discussion}

Notre travail montre l'évolution spectaculaire de l'incidence du DT1 de l'enfant enregistré pour la wilaya d'Oran ces 25 dernières années. Cette évolution est mondialement constatée avec des accroissements importants dans les pays émergents, lorsqu'on compare les chiffres des enquêtes des organismes internationaux effectuées avant et après l'an- 
née 2000, avec de plus des bouleversements du classement par pays. On note ainsi dans l'Atlas du Diabète de la FID 2017 [2] le maintien dans le 'top 10' de la Finlande (en 1 ère position), du Koweit (2ème), de la Suède (3ème), de la Norvège (5ème), du Royaume Uni (8ème), de l'Irlande (9ème) et du Danemark (10ème) [2] qui figuraient déjà dans les 10 premiers du classement du Diamond Project Group [6] qui avait fait le point des incidences 1990-1999 de 57 pays participants, cependant que font leur irruption dans l'Atlas, l'Arabie Saoudite (4ème), l'Algérie (6ème) et le Maroc (7ème). Ces nouveaux promus sont représentés par un pays Moyen-oriental, l'Arabie Saoudite qui vient de publier les relevés de son registre en 2017 [7] comme le Koweit [8] et par deux pays Nord-africains, l'Algérie et le Maroc dont les données, pour ce dernier, ont été extrapolées à partir des données algériennes [2]. Cette évolution entre les deux enquêtes relativise la notion classique des gradients Nord-Sud et Ouest-Est, les pays du Sud et d'Orient étant de plus en plus impactés par l'épidémie mondiale du DT1.

Les raisons de cette véritable épidémie mondiale font appel à plusieurs théories et hypothèses étio-pathogéniques. Les facteurs de risque du DT1 évoqués sont avant tout environnementaux de nature climatique, géographique, sociaux-démographiques, alimentaires et infectieux car l'augmentation de l'incidence du DT1 est apparue trop rapidement pour dépendre de la génétique classique, mais laisse toutefois place à des mécanismes épi-génétiques [9]. Nous rappellerons la théorie hygiéniste qui stipule que des déséquilibres immunitaires pourraient être induits par la diminution de l'exposition aux infections communautaires par le fait des progrès de l'hygiène générale et de la pratique des vaccinations [10]. La théorie dite de l'accélération, en appelle à l'épidémie du surpoids et de l'obésité qui se développe parallèlement au DT1 chez l'enfant [11,12,13]. De fait, dans le recrutement présenté nous constatons cette dernière relation qui pourrait expliquer à tout le moins le recul de l'âge de début du DT1, mais probablement aussi une partie de l'augmentation de l'incidence globale du diabète par le biais de la résistance à l'insuline secondaire à l'obésité [14]. En faveur de cette hypothèse, s'ajoutent la description de formes intermédiaires de diabète de l'enfant, dénommés «diabètes doubles» et l'existence des cas de DT1 de type 2 à partir de l'âge de 10 ans qui posent de plus des problèmes de diagnostic différentiels assez ardus avec le type 1 [15]

Nous avons constaté une augmentation constante des cas incidents du DT1 rapportés à leurs années de naissance, qui démontre une augmentation vraie de l'incidence du DT1 de l'enfant et non un simple effet de la translation de l'âge de début. Ce qui permet aussi de faire une projection sur les années à venir, comme l'ont montré d'autres auteurs et de pronostiquer d'éventuels ralentissements à venir. Berhan en Suède [16] étudiant l'évolution de l'incidence du DT1 dans des cohortes d'année de naissance, montre que l'incidence du DT1 augmente dans les cohortes des plus jeunes de 1978 à 1999 pour ralentir dans les années de naissance 1999-2002 et 2003-2006, ce qui indique un changement d'exposition aux facteurs de risque qui affectent les enfants les plus jeunes. Ceci peut s'expliquer par les efforts fournis en Suède, pour diminuer l'obésité infantile par un programme national de Santé publique appliqué au niveau de la lutte contre l'obésité. Ce n'est pas le cas de notre cohorte des moins de 15 ans qui voit son nombre par année de naissance continuer de progresser d'une année de naissance sur l'autre, sans l'espoir raisonnable d'un prochain plateau d'incidence, les années après 2002, non saturées (en rouge) dépassant déjà celles saturée d'avant 2002 (figure 4). Les projections EURODIAB pour 2025 n'incitent pas à l'optimisme [17]

Conséquemment le recul de l'âge de début déjà évoqué par notre équipe $[18,19]$ implique l'apparition en milieu pédiatrique de complications âges-dépendantes, la néphropathie diabétique en premier lieu, qui posent la question du milieu médical approprié pour leur prise en charge [20].

La prévalence augmente logiquement vers la classe d'âge [10-14] ans par effet de cohorte ce qui va poser les problèmes liés à la transition de la médecine d'enfants vers la médecine d'adultes qui iront fatalement en s'amplifiant et nécessiteront des dispositions spécifiques [21]. Le nombre total des DT1 des moins de 20 ans diagnostiqués par pays en 2017 range notre pays à la 6ème place avec 42500 DT1 derrière les USA, l'Inde, le Brésil, la Chine et la Russie [2] mettant en évidence la disproportion des moyens médicaux qui mettent à mal les pays à moyen ou faible revenu.

En Algérie, les différentes études d'incidence réalisées vont dans le même sens que la nôtre. A l'Est, dans la wilaya de Constantine, Bouderda rapporte l'augmentation de l'incidence annuelle moyenne pour 100000 de moins de 15 ans qui est passée de 9,57 en 1990-1994 à 14,33 en 2000-2004 $[22,23]$. Pour les mêmes périodes, nos chiffres font état de 7,82 et de 14,20, respectivement. Au Centre du pays, d'après le registre de la wilaya d'Alger, les chiffres étaient de 22,8 p.100 000 entre 2009 et 2010 et de 29,35 en 2016 [24], très proches de nos propres chiffres qui font état de 21,2 en 2009-2010 et de 32,98 en 2016.

En ce qui concerne la wilaya d'Oran, on peut encore ajouter que notre registre a donné lieu à une thèse en épidémiologie et génétique [25], à une première publication en 1990 [26] et a été pris en compte par le projet Diamond pour situer l'Algérie dans l'enquête 90-99 au 29ème rang sur 57 pays participants avec une incidence considérée alors comme intermédiaire [6]. On parlait déjà d'épidémie mondiale de diabète de l'enfant, dont on peut remarquer le stigmate avec une première alerte significative entre 1985 
et 1990 (Figure 2).

Concernant le sex-ratio, les données d'Eurodiab [27] et bien d'autres depuis révèlent dans le DT1 un sex-ratio (garçon/ fille) non significativement différent de celui de la population générale considérée, comme nous le constatons nousmêmes si l'on considère la totalité de nos DT1. Nous n'avons pas d'explication satisfaisante pour ce qui est des fluctuations importantes du sex-ratio de notre recrutement dans le temps (Tableau 2).

Tableau 2. DT1 de l'enfant de la wilaya d'Oran. Evolution et comparaison du sex-ratio de 1973-2017, par périodes remarquables

\begin{tabular}{|l|c|c|c|c|c|}
\hline \multicolumn{1}{|c|}{ Périodes } & $1973-1977$ & $1978-1997$ & $1998-2007$ & $2008-2017$ & $1973-2017$ \\
\hline Garçons & 99 & 145 & 328 & 603 & 1175 \\
\hline Filles & 100 & 182 & 287 & 614 & 1183 \\
\hline $\begin{array}{l}\text { Sex-ratio } \\
\text { (G.IF.) }\end{array}$ & 0,99 & 0,8 & 1,14 & 0,98 & 0,99 \\
\hline \multicolumn{1}{|c|}{ P* $^{*}$} & & $=0,24$ & $<0,02$ & $=0,15$ & \\
\hline
\end{tabular}

Le caractère saisonnier au moment du diagnostic du DT1 suit un modèle sinusoïdal confirmé par Eurodiab [27] et par les auteurs finlandais [28] qui ont montré l'existence de la prééminence hivernale dans tous les pays d'Europe, pour les deux sexes et pour les trois classes d'âge entre 0 et 14 ans. Ce profil a été mis en rapport avec les infections virales saisonnières capables de déclencher une nouvelle poussée auto-immune et de précipiter la carence en insuline.

Concernant notre propre recrutement, cette saisonnalité reste valable et significative jusqu'en 2014, année après laquelle la significativité disparaît (Tableaux 3 et 4). Cette constatation, non documentée dans la littérature, hormis le même constat relevé dans le registre de la wilaya d'Alger en 2016 [23] avec 48,2\% en automne-hiver et $51,8 \%$ en printemps-été $(p=0,23)$ pour 262 nouveaux cas, renforçant nos propres chiffres 2016 de $49,7 \%$ et $50,3 \%$ pour chacune des 2 périodes, froide et chaude, respectivement, pour 159 nouveaux cas. Ceci plaide en faveur du rôle maintenant prégnant de l'environnement non infectieux, par rapport à sa composante infectieuse jusque là dominante.

Ce phénomène probablement exacerbé dans notre pays qui a connu des bouleversements relativement tardifs et très brutaux en termes d'ouverture à la mondialisation et notamment en matière alimentaire, maintenant calqué sur le modèle occidental.
Tableau 3. Saisonnalité des nouveaux cas de DT1 de l'enfant de la wilaya d'Oran de 1973 à 2017. Comparaison des répartitions mensuelles cumulées des périodes 1973-2014 et 2015-2017

\begin{tabular}{|c|c|c|c|c|c|c|c|c|c|c|c|c|c|}
\hline & Jan. & Fév. & Mar. & Avr. & Mai & Juin & Juil. & Août & Sept. & Oct. & Nov. & Déc. & $P^{*}$ \\
\hline $\begin{array}{c}1973- \\
2017\end{array}$ & 213 & 188 & 200 & 152 & 160 & 159 & 134 & 155 & 174 & 207 & 205 & 242 & $<0,01$ \\
\hline $\begin{array}{c}1973- \\
2014\end{array}$ & 183 & 157 & 147 & 120 & 123 & 119 & 103 & 102 & 129 & 158 & 164 & 180 & $<0,01$ \\
\hline $\begin{array}{c}2015- \\
2017\end{array}$ & 30 & 31 & 53 & 32 & 37 & 40 & 31 & 53 & 45 & 49 & 41 & 62 & $=0,58$ \\
\hline
\end{tabular}

*Comparaison des rangs des mois froids (J., F.,M.,O.,N.,et D.) et des mois chauds (A.,M.,J.,J.,A. et $S$;) , test de Wilcoxon.

Tableau 4. Saisonnalité des nouveaux cas de DT1 de l'enfant de la wilaya d'Oran de 1973 à 2017. Comparaison des proportions Hiver/Eté des périodes 1973-2014 et 2015-2017

\begin{tabular}{|c|c|c|c|}
\hline & Saison froide & Saison chaude & Total \\
\hline $1973-2014$ & $989(58,69 \%)$ & $6 \%(41,3190)$ & $1685(100 \%)$ \\
\hline $2014-2017$ & $266(52,78)$ & $238(47,220)$ & $504(100 \%)$ \\
\hline
\end{tabular}

\section{Conclusion}

La tenue du registre DT1de l'enfant de la wilaya d'Oran nous a permis d'évaluer son incidence et sa progression dans le temps qui risque de perdurer. L'âge du diagnostic se décale très majoritairement au-dessous de 10 ans, compliquant encore le travail des équipes médicales et des familles.

Les causes intimes de cette progression du DT1 sont difficiles à identifier et font l'objet de recherches intensives. L'identification des facteurs environnementaux précipitant permettront à tout le moins d'envisager une prévention primaire.

\section{Conflits d'intérêt}

Les auteurs déclarent n'avoir aucun conflit d'intérêt.

\section{Références bibliographiques}

[1]. W 1. Barat P. Augmentation de l'incidence du diabète de type 1 chez les enfants les plus jeunes : quelles conséquences pour les pédiatres? Arch Pediatr 2014, 21: 449-451.

[2]. Atlas du diabète de la FID.8ème édition 2017.

[3].Patterson C, Guariguata L, Dahlquist G et al. Diabetes in the young - a global view and worldwide estimates of numbers of child ren with type 1 diabetes. Diabetes Res Clin Pract 2014; 103: 161-175. 
[4].World Health Organization. Definition and diagnosis of diabetes mellitus and intermediate hyperglycemia : report of a WHO/IDF consultation. World Health Organization, 2006.

[5]. American Diabetes Association. Classification and Diagnosis of Diabetes. Diabetes Care 2017; 40 (Supplement 1): S11-S24.

[6].Bruno G, Runzo C, Cavallo-Perin DIAMOND Project Group. Incidence and trends of childhood type 1diabetes worldwide 1990-1999. Diabet Med 2006; 23: 857-66.

[7]. Alotaibi A, Perry L, Gholizadey L, Al-Ghanmi A. Incidence and prevalence rate of diabetes diabetes in Saudi Arabia : An overwiew. J Epidemiol Global Healt 2017; 7: 211-218

[8].Shaltout AA, Wake D, Thanaraj TA et al. Incidence of type 1 diabetes has doubled in Kuwaiti children 0-14 years over the last 20 years. Pediatr Diabetes 2017; 18: 761-766.

[9].Jerram ST, Dang MN, Leslie RD. The Role of Epigenetics in Type 1 Diabetes. Curr Diab Rep. 2017; 17: 89.

[10].Bach JF, Chatenoud L. The hygiene hypothesis: an explanation for the increased frequency of insulin-dependent diabetes. Cold Spring Harb Perspect Med. 2012; 2(2): a007799.

[11]. Betts P, Mulligan J, Ward P, Smith B, Wilkin T. Increasing body weight predicts the earlier onset of insulin-dependant diabetes in childhood: testing the 'accelerator hypothesis' . Diabet Med. 2005; 22(2): 144-151.

[12].Kibirige M, Metcalf $B$, Renuka R, Wilkin TJ. Testing the accelerator hypothesis: the relationship between body mass and age at diagnosis of type 1 diabetes. Diabetes Care. 2003; 26(10): 2865-2870.

[13].Evans JM, Newton RW, Ruta DA, et al. Socio-economic status, obesity and prevalence of Type 1 and Type2 diabetes mellitus. Diabet Med J Br Diabet Assoc 2000; 17: 478-480.

[14].Touhami M. Validation de l'hypothèse de l'accélération comme facteur de précocité et d'augmentation d'incidence du DT1 de l'enfant sur la base d'un enregistrement de 42 ans (1975-2016). CO-031. Congrès de la SFD Lille 2017.

[15]. Largay J. Case Study: New-Onset Diabetes: How to Tell the Difference Between Type 1 and Type 2 Diabetes. Clin Diabetes 2012; 30: 25-26.

[16].Berhan $Y$ et al. Thirty Years of Prospective Nationwide Incidence of Chilhood Type 1 Diabetes: The Accelerating Increase by Time Tends to Level Off in Sweden. 2011; 60: 577-581.

[17].Maahs DM, West NA, Lawrence JM, et al. Epidemiology of Type1 Diabetes. Endocrinol Patterson CC, Dahlquist GG, Gyurus E, et al. EURODIAB Study Group. Incidence trends for childhood type 1 diabetes in Europe during 1989-2003 and predicted new cases 2005-20: a multicentre prospective registration study. Lancet 2009; 373: 2027-2033.

[18].Niar S. Epidémiologie du diabète de type 1 chez l'enfant et l'adolescent diabétique de l'Ouest-algérien. Thèse de Doctorat en Sciences Médicales. Faculté de médecine d’Oran, année 2012.
Epidémiologie générale du diabète de type 1 chez l'enfant: Le registre de la wilaya d'Oran 1973-2017

[19].Niar S, Naceur M, Bessahraoui M, Bouchetara A, Zennaki A, Bouziane-Nedjadi $K$, Touhami $M$. Épidémiologie du diabète de type 1 de l'enfant dans le département d'Oran (Algérie) de 1975 à 2014 Med Mal Metabol 2015; 9(5): 529-532.

[20]. Raile K, Galler A, Hofer S, Herbst A, Dunstheimer D, Busch P, Holl RW. Diabetic nephropathy in 27,805 children, adolescents, and adults with type 1 diabetes: effect of diabetes duration, A1C, hypertension, dyslipidemia, diabetes onset, and sex. Diabetes Care. 2007 ; 30(10): 2523-2528.

[21]. Jacquin P, Loison S, Le Roux E, Rocaboy C, Guilmin-Crépon S, Tubiana-Rufi N, Groupe Collaboratif «Pass'Âge». Adolescents et jeunes adultes atteints de diabète : réussir la transition de la pédiatrie à la médecine d'adulte. Médecine des maladies Métaboliques 2015; 9 : 661-667.

[22].Bouderda Z. Epidémiologie du diabète de type 1 de l'enfant dans l'Est algérien. Thèse de Doctorat en Sciences Médicales. Faculté de médecine de Constantine, année 2000

[23]. Bouderda Z, Bouchair N, Boumaaraf N. Incidence du diabète de l'enfant à Constantine, 1990-2004. Arch Pédiatr 2008 ; 15(5): 961

[24]. INSP. Registre du diabète de type 1chez l'enfant âgé de moins de 15 ans au niveau de la wilaya d'Alger. Année 2016.

[25]. Bessaoud K. Epidémiologie et génétique du diabète insulinodépendant du diabète dans l'Ouest-algérien. Thèse de Doctorat en Sciences Médicales. Faculté de médecine d’Oran, année 1986.

[26].Bessaoud K, Boudraa G, Deschamps I, et al. Épidémiologie du diabète insulino-dépendant juvénile en Algérie (Wilaya d'Oran). Rev Épidémiol Santé Publique 1990; 38: 91-9.

[27]. Bruno G, Runzo C, Cavallo-Perin DIAMOND Project Group. Incidence and trends of childhood type 1diabetes worldwide 1990-1999. Diabet Med 2006; 23: 857-866.

[28].Moltchanova EV, Schreier N, Lammi N, Karvonen M. Seasonal variation of diagnosis of Type 1 diabetes mellitus in children worldwide. Diabet Med. 2009 , 26(7): 673-678. 
